\title{
COMPARING PERFORMANCE WHEN USING A NEW STYLE LARGE TOUCHSCREEN COMPARED TO A TRADITIONAL IN-VEHICLE TOUCHSCREEN
}

\author{
Timothy Brown ${ }^{1}$, Dawn Marshall ${ }^{1}$, Neil Lerner ${ }^{2}$ \\ ${ }^{1}$ National Advanced Driving Simulator, University of Iowa, Iowa City, IA, USA \\ ${ }^{2}$ Westat, Rockville, MD, USA \\ Email: timothy-1-brown@uiowa.edu
}

\begin{abstract}
Summary: New in-vehicle touch screen displays are increasing in size and complexity, and the effect on distraction to the driver associated with their use is unclear. Large touchscreen displays, such as those in the Tesla, provide a richer display environment as well as a larger area compared to traditional in-vehicle touchscreens even when the same capabilities are available. This simulator study examines how performing the same tasks on two different types on in-vehicle displays impacts glance behavior, vehicle control and workload. Results show that the large touchscreen results in longer average glance lengths, a greater percentage of glances of more than 2-seconds, but fewer glances. For vehicle control, there were no differences in lateral control, but the large touchscreen showed less variability in speed and speed range overall, but not uniformly across the tasks. Drivers did not report different levels of workload between the two interfaces. The results point to the need for careful design to minimize the likelihood of long glances as vehicle design moves to larger displays.
\end{abstract}

\section{OBJECTIVE}

The increasing pace of technological advancement continues to make new interfaces available for use in vehicles. These technologies are often developed for different areas and the impact on drivers is not well understood. They may change inaction with the vehicle in ways that impact ease of use, workload, distraction and safety. Systems such as voice interface offer the hope of eliminating eyes-off-road time while engaging with the vehicle, but may increase workload or increase the time required to complete a task. Similarly, new touchscreens that provide more screen space may allow for more aesthetic designs or greater customizability, but the impact of these richer displays is not well understood in terms of distraction potential.

\section{Distraction}

Driver distraction is a significant traffic safety problem. According to the National Highway Traffic Safety Administration (NHTSA), distracted driving was the cause of 3,450 of the 37,461 fatalities $(9.2 \%)$ in motor vehicle crashes in the United States in 2016 (National Highway Traffic Safety Administration, 2017). The 100-car naturalistic driving study found the that distraction and inattention were a contributing factor in about $80 \%$ of crash and near-crash events (Klauer, Dingus, Neale, Sudweeks, \& Ramsey, 2006), while a separate study focused on front-to-rear end crashes found that $75 \%$ of novice drivers were distracted leading up to the crash (Carney, Harland, \& McGehee, 2016). More recent naturalistic work has shown that distraction significantly increases the risk of crash (Klauer, Ehsani, McGehee, \& Manser, 2015)

The effort to combat driver distraction in the United States has been ongoing with efforts at the state and federal level. At the state level, changes in state laws have been put in place to more easily allow for enforcement of dangerous behavior that increases risk. Distraction in the driving environment can take many forms from distraction external to the vehicle (Stutts et al., 2003; 
Wallace, 2003), passengers (Williams, Ferguson, \& McCartt, 2007), mind wandering (Yanko \& Spalek, 2014), mobile devices (Centers for Disease Control and Prevention, 2013), and invehicle systems (Lee, Brown, Caven, \& Haake, 2000). In 2012, NHTSA issued guidelines for identifying in-vehicle visual-manual systems that might cause distraction for the driver to aid OEMs in the design of their interfaces (Department of Transportation, 2012). These guidelines focus on reducing distraction by having systems designed in such a way as to have average glance times of less than two-seconds, the total eyes-off-road time of less than twelve-seconds, and less than $15 \%$ of the glances being longer than two-seconds.

\section{Touchscreens}

Touchscreen have grown in popularity since their use became widespread in mobile devices such as phones and tablets. The use of touchscreens in vehicles was first introduced in the 1986 Buick Riviera, and has recently seen rapid and steady increases in use to the point that they are present in most new vehicles manufactured. There remain differences in uses between manufacturers and new methods for interacting with the displays. Despite their growing prevalence, their introduction has not been without problems. As touchscreens have replaced physical controls there has been criticism that the new approach is inferior to older technology in that it increases driver distraction by requiring the driver to take their eyes off the road to be used (e.g., Then, 2015). Additionally, pushback by consumers has even caused at least one manufacturer to reintroduce more traditional buttons to augment touchscreen controls (Davis, 2013).

In summarizing the potential human factors issues associated with touch screens, much of the research has focused on the interactions with the screen in terms of input methods such as general methods for scrolling (Kujala, 2013), haptic/vibrotactile feedback (Gaspar, 2011), and Swype inputs (Harvey, Stanton, Pickering, McDonald, \& Zheng, 2011). A great deal of research exists on the use of the touchscreen in cars. Much of the new research focuses on different methods of interacting with the touchscreen to reduce distraction and improve performance relative to the direct touch method. However, little published research (Rümelin \& Butz, 2013) to date has tackled the issues associated with larger touchscreen displays in terms of scanning and interaction while driving.

\section{METHODS}

\section{Participants}

A total of eight participants divided into four age bins (19-24, 35-39, 40-54 and 55-75) balanced between males and females in each age bin participated in this research. Participants were active drivers with a valid license, driving at least 3000 miles per year and experience using a cell phone while driving. This research was conducted with approval of the University Iowa Institutional Review Board (FWA00003007).

\section{Apparatus}

Testing was conducted using a miniSim ${ }^{\mathrm{TM}}$ driving simulator and a Dikablis eyeglasses professional for eye-tracking. . The simulator was configured to the specifications outlined in the NHTSA Visual-Manual Distraction Guidelines (National Highway Traffic Safety Administration, 2013). The simulator has a horizontal forward field of view of 31.3 degrees, at a distance of 2.0 meter from the driver's eye point to the display screen with a screen resolution of less than 3 arc minutes per pixel, and a distance of 2.0 meters ( 78.7 inches). The display interface was positioned similar to that of a production vehicle so that the eye glances and reach distances to the tasks were realistic. Driving consisted of driving behind a lead vehicle in a car following 
task which provided participants a reason to glance back to the roadway while engaged in a task. Each participant was given a short period to practice before beginning task trials. Data for each of the tasks were collected in a separate drive. The Dikablis eye-tracker is a head-mounted system that collects data at $60 \mathrm{~Hz}$ with accuracy of $0.1^{\circ}-0.3^{\circ}$ of visual angle.

\section{Experimental Design}

Data was collected for three interfaces: a traditional touchscreen, a large touchscreen, and gesture system. The analysis for this paper focuses on only the two touchscreen interfaces. Across each of the systems, participants completed a series of 12 representative tasks. These tasks were selected from phone use, navigation, media and radio to conform to the NHTSA Visual-Manual Guidelines. Three tasks were selected from each category. This resulted a completely within subjects $3 \times 12$ design. This resulted in 8 data points for each task within each system and 96 data points per system when collapsing across tasks.

\section{Interfaces}

The two interfaces were provided on the same display, a Microsoft Surface Book, with a screen size of 13.5 inches (diagonal) located to the right of the driver. The traditional touchscreen interface $(6.25 " \mathrm{~W} \times 3.75$ " $\mathrm{H}$ ) was designed to be similar to those currently used in many production models (see Figure 1) and included no hard buttons. The large touchscreen interface (11.3" W x 7" H) was designed to include a surface area similar to vehicles such as the Tesla and was designed to mimic the richer interfaces that tend to be found on these newer displays (see Figure 2) and included no hard buttons.

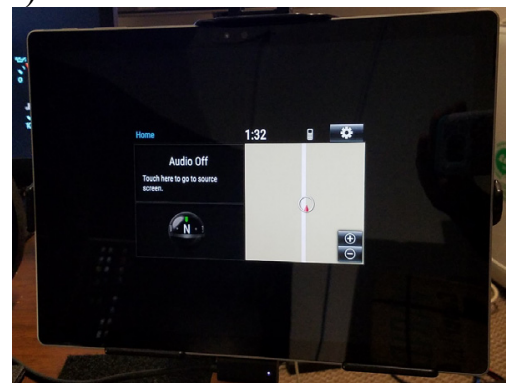

Figure 1. Home screen for traditional interface

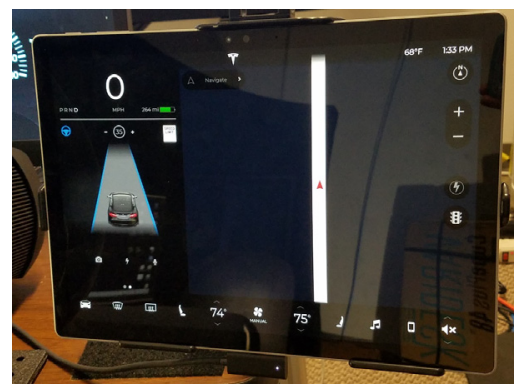

Figure 2. Home screen for large screen interface

\section{Tasks}

Tasks were selected such that they would be representative and provide a similar number of steps across the interfaces tested. Tasks were included for CD usage (select album, select track, choose album), map (zoom in, zoom out, identify heading), phone (call recent, call from contacts, 10digit dial), and radio (select from preset, tune station, select from preset list).

\section{Procedure}

Participants were screened by phone to verify eligibility. Upon arrival, the study was explained and informed consent was obtained. Participants were then trained on the study procedures. Data was collected for each task for each interface using both an occlusion method and using the driving simulator. The occlusion method, which involved performing the tasks in fixed windows of time without driving, preceded driving simulator method and is not analyzed as it is outside the scope of the research questions. The order of the interfaces was randomized. Participants were trained on each task before data was collected and needed to be able to complete the task successfully three times without error. During the simulator drive, participants were asked to complete each task one time while verbalizing the end of the task with "done" and a workload scale score. 


\section{Measures}

Dependent measures for this analysis were selected to assess the impact of display type on distraction and driving performance. Measures can be divided into three categories: glance behavior, driving performance, and workload. Glance data was assessed by measuring the glance to toward the display while completing the task. This provides measures of the number of glances, total glance time to the display, mean glance duration, and percent of glances longer than 2 seconds. Driving data was collected at $30 \mathrm{~Hz}$ and reduced to provide analyzable measure of performance. These measures included standard deviation of speed, delta speed (difference between beginning and ending speed during the task), speed range (difference between maximum and minimum speed during the task), lane departures defined as part of the vehicle being outside the lane, and standard deviation of lane position. Workload was assessed using the Instantaneous Self-Assessment of workload technique (Brennan, 1992). This scale uses rankings of 1 to 5 with level 1 corresponding to under-utilized, level 2 as relaxed, level 3 as comfortable busy pace, level 4 as high and level 5 as excessive.

\section{RESULTS}

The SAS General Linear Models (GLM) procedure was used to perform an Analysis of Variance (ANOVA) on the parametric dependent measures. To assess the impact of different display types on driver interaction, the analysis will be divided into three parts: glances to the display, driving performance (lateral and longitudinal), and perceived workload.

When looking at the glance behavior, significant differences are observed in the number of glances $(F(1,7)=16.09, p=0.0051)$, mean glance duration $(F(1,7)=13.46, p=0.0080)$ and percent long glances $(F(1,7)=6.45, p=0.0387)$. As can be observed in Figure 3, when using the traditional display, drivers had on average 4.9 glances to the display, but with the larger display had 3.6 glances. The duration of these glances was on average 1.25 seconds for the traditional display and 1.53 seconds for the large display (see Figure 4). Additionally, the percent of the glances that were longer than 2 seconds was $6.6 \%$ for the traditional display and $19.0 \%$ for the large display (see Figure 5). No differences were observed for total glance duration ( $\mathrm{p}>0.05)$. When looking at the driving data, significant differences were observed for the longitudinal measures of standard deviation of speed $(F(1,7)=14.92, p=0.0062)$ and speed range $(F(1,7)=13.36, p=0.0081)$. Both measures had significant interactive effects with the task $(\mathrm{F}(1,76)=2.71, \mathrm{p}=0.0053$ and $\mathrm{F}(1,76)=2.69, \mathrm{p}=0.0056$, respectively). The interactive effects are plotted in Figure 6 for standard deviation of speed and Figure 7 for speed range. For both measures, the traditional display results in greater values for 9 out of 12 tasks. In general, speed variability was greater by 0.25 mph and speed range was greater by $0.85 \mathrm{mph}$ with the traditional display; however, as can be seen by the figures, it was not uniform across tasks. There were no additional differences in driving performance for delta speed, standard deviation of lane position or lane departures $(p>0.05)$. When looking at perceived workload, there was no differences in between the displays $(p>0.05)$ despite significant differences between tasks.

\section{LIMITATIONS}

It should be noted that the research includes a limited number subjects, and only a sample of the types of tasks that can be performed that cannot guarantee broad generalizability. Display type is a compound variable as in includes both size and style of interface and differences cannot be attributed definitively to either difference. Additionally, it should be noted that the horizontal field-of-view, while sufficient for the car following task, was small relative to horizontal field of view of larger simulators and cars and may have had unknown effects on glance behavior. 


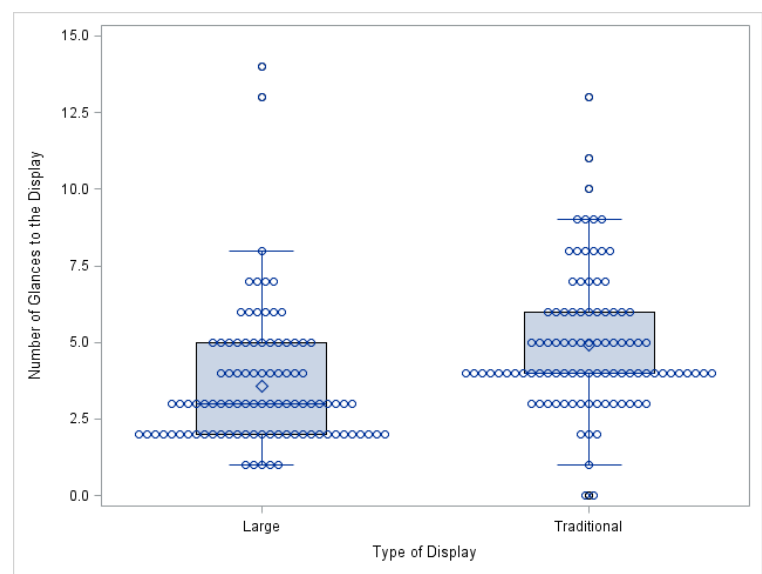

Figure 3. Number of Glances to the Display.

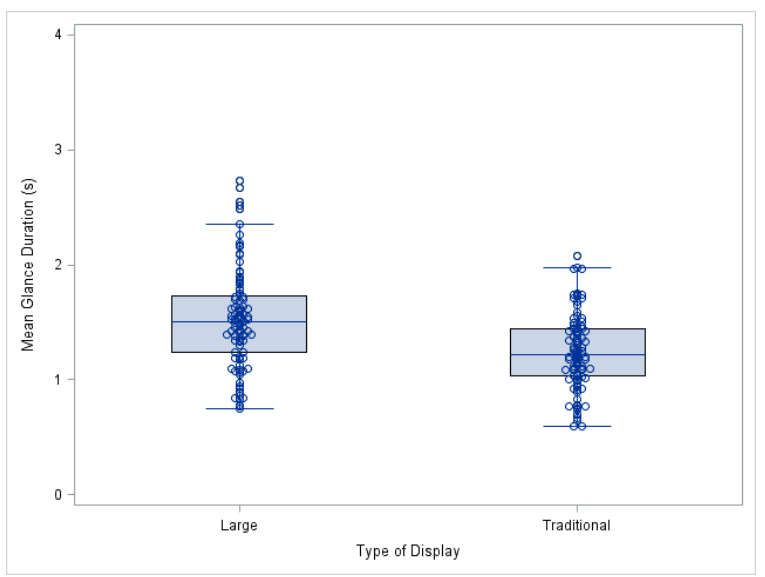

Figure 4. Average Glance Duration to the Display

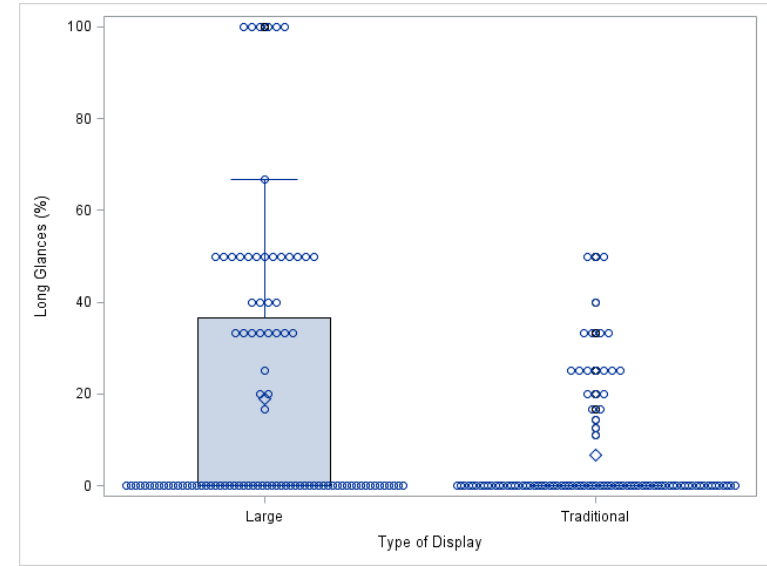

Figure 5. Percent of the Glance that were more than 2 Seconds.

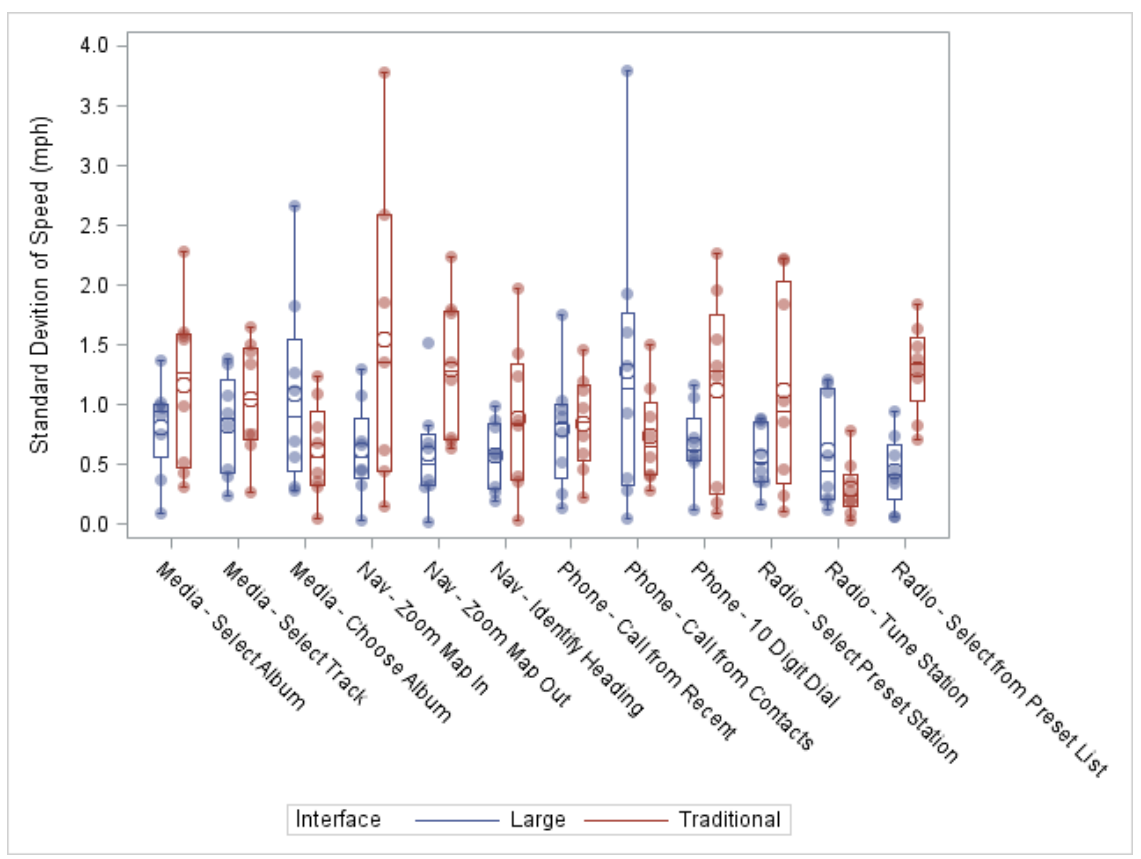


Figure 6. Variability in Speed.

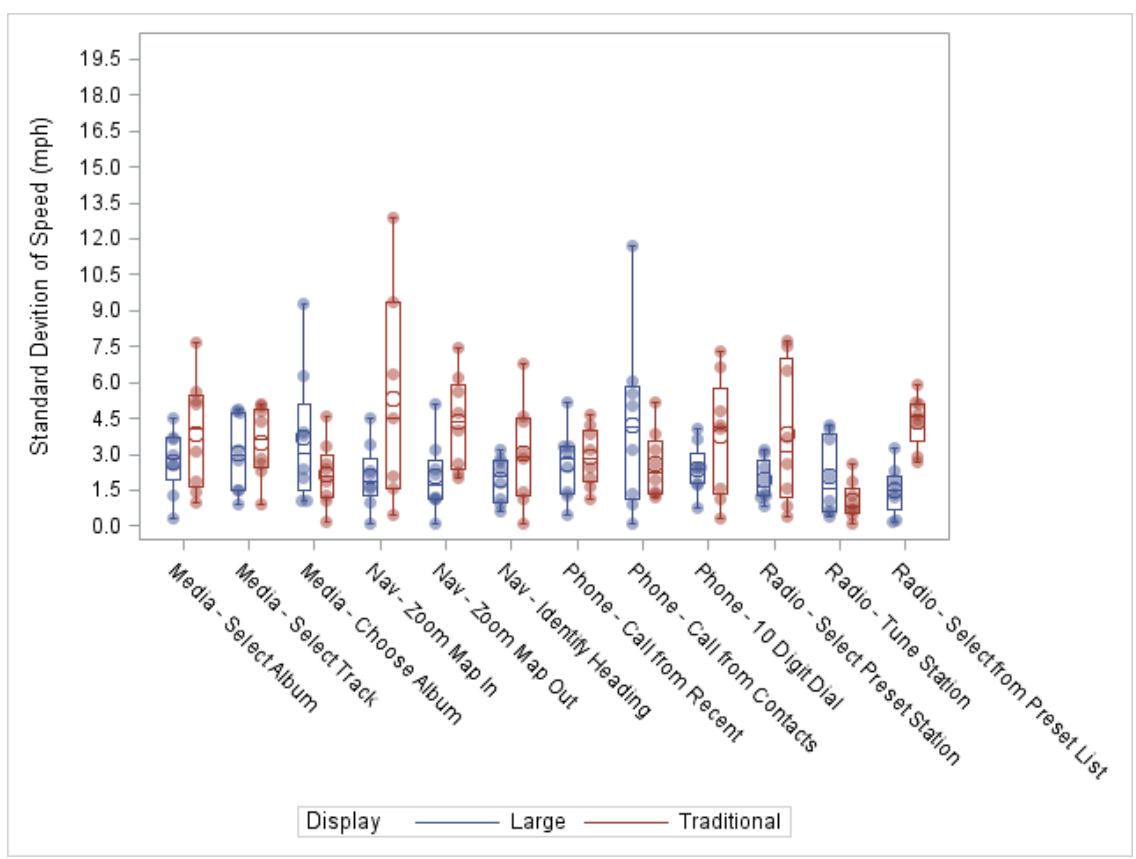

Figure 7. Range of Speed.

\section{CONCLUSIONS}

In considering the results, it is clear that type of display has an impact how drivers interact with it. The large display results in longer and less frequent glances away from the road. The result of these more frequent glances and a general degradation in longitudinal control as evidenced by less precise speed maintenance while engaged in the task. An interesting component of this is that despite differences between the systems, the drivers do not appear to sense a significant difference in workload. The challenge in considering the displays is to balance the proportion of longer glances associated with the larger display with the more frequent shorter glances that are associated with more precise speed control. Absent the greater proportion of long glances, it would be easy to make an argument these displays providing a better user interface; however the problem with longer glances is the increased chance of something changing in the driving environment that could lead to crashes. Perhaps in the way designed, the larger touchscreen interface is superior. Care should be taken in designing larger touchscreens to not encourage even longer glances that may produce safety disbenefits. Overall, larger and richer displays can provide an alternative to the smaller displays that provide designers with greater flexibility while reducing distraction and its related effects if appropriately designed.

\section{ACKNOWLEDGMENTS}

This research was funded by the NHTSA under contract DTNH2211D00237L/0016. The opinions presented are those of the authors and not necessarily those of NHTSA. We thank Patrick McGill for his efforts in collecting data for this project.

\section{REFERENCES}

Brennan, S. (1992). An experimental report on rating scale descriptor sets for the instantaneous self assessment (ISA) recorder. Portsmouth: DRA Maritime Command and Control Division. DRA Technical Memorandum (CAD5), 92017. 
Carney, C., Harland, K. K., \& McGehee, D. V. (2016). Using event-triggered naturalistic data to examine the prevalence of teen driver distractions in rear-end crashes. Journal of Safety Research, 57, 47-52.

Centers for Disease Control and Prevention. (2013). Mobile device use while driving--United States and seven European countries, 2011. MMWR. Morbidity and mortality weekly report, 62(10), 177.

Davis, C. (2013). Ford bringing back buttons after touchscreen distraction fears. Slash Gear. Retrieved from Slash Gear website:

Department of Transportation. (2012). Visual-Manual NHTSA Driver Distraction Guidelines for In-Vehicle Electronic Devices. Washington, DC

Gaspar, A. A. (2011). Design and evaluation of haptic feedback for in-vehicle touch screen.

Harvey, C., Stanton, N. A., Pickering, C. A., McDonald, M., \& Zheng, P. (2011). To twist or poke? A method for identifying usability issues with the rotary controller and touch screen for control of in-vehicle information systems. Ergonomics, 54(7), 609-625.

Klauer, S. G., Dingus, T. A., Neale, V. L., Sudweeks, J. D., \& Ramsey, D. J. (2006). The impact of driver inattention on near-crash/crash risk: An analysis using the 100-car naturalistic driving study data (DOT HS 810 594). Retrieved from Washington, DC:

Klauer, S. G., Ehsani, J. P., McGehee, D. V., \& Manser, M. (2015). The effect of secondary task engagement on adolescents' driving performance and crash risk. Journal of Adolescent Health, 57(1), S36-S43.

Kujala, T. (2013). Browsing the information highway while driving: three in-vehicle touch screen scrolling methods and driver distraction. Personal and Ubiquitous Computing, 17(5), 815-823.

Lee, J. D., Brown, T. L., Caven, B., \& Haake, S. (2000). Speech-based Interaction with Invehicle Computers: The Effect of Speech-Based E-mail on Drivers'Attention to the Roadway. Paper presented at the NHTSA Driver Distraction Internet Forum.

National Highway Traffic Safety Administration. (2013). Visual-Manual NHTSA Driver Distraction Guidelines for In-Vehicle Electronic Devices (NHTSA-2010-0053). Retrieved from

National Highway Traffic Safety Administration. (2017). Fatal Motor Vehicle Crashes: Overview (DOT HS 812 456). Retrieved from

National Highway Traffic Safety Administration. (2018). U.S. DOT and NHTSA Kick Off 5th Annual U Drive. U Text. U Pay. Campaign [Press release]

Rümelin, S., \& Butz, A. (2013). How to make large touch screens usable while driving. Paper presented at the Proceedings of the 5th International Conference on Automotive User Interfaces and Interactive Vehicular Applications.

Stutts, J., Feaganes, J., Rodgman, E., Hamlett, C., Meadows, T., Reinfurt, D., . . Staplin, L. (2003). Distractions in everyday driving. Retrieved from

Then, E. (2015). The Problm with Tocuh Controls in the Automotive Industry. Slash Gear.

Retrieved from Slash Gear website:

Wallace, B. (2003). External-to-vehicle driver distraction: Scottish Executive, Social Research.

Williams, A. F., Ferguson, S. A., \& McCartt, A. T. (2007). Passenger effects on teenage driving and opportunities for reducing the risks of such travel. Journal of Safety Research, 38(4), 381-390.

Yanko, M. R., \& Spalek, T. M. (2014). Driving with the wandering mind: the effect that mindwandering has on driving performance. Human Factors, 56(2), 260-269. 